\title{
Quantum wires and dots induced in a semiconductor by charged metallic filaments separated by an isolating barrier
}

\author{
Z. S. Gribnikov ${ }^{\text {a) }}$ \\ Institute of Quantum Sciences, Michigan State University, East Lansing, Michigan 48824 \\ and Department of Electrical Engineering and Computer Science (EECS), University of Michigan, Ann \\ Arbor, Michigan 48109 \\ G. I. Haddad \\ Solid-State Electronic Laboratory, Department of Electrical Engineering and Computer Science (EECS), \\ University of Michigan, Ann Arbor, Michigan 48109
}

(Received 18 October 2004; accepted 22 November 2004; published online 6 January 2005)

\begin{abstract}
A very thin positively charged metallic filament separated from a surface of a semiconductor $(S)$ by a thin nontunneling potential barrier $(B)$ induces a quantum wire $(\mathrm{QWr})$ in the semiconductor at the $B / S$ interface. Single-electron quantum states of this QWr are controlled by a potential (and a charge) of the metallic filament. Two close parallel metallic filaments placed over such a $B / S$ interface form a double-quantum wire with the ground and the first excited electron states, which appear as a result of a symmetric-antisymmetric splitting of the ground electron state in the single QWr. Two crossed metallic filaments, which are parallel to the $B / S$ interface, form a quantum dot with completely localized electron states under the crossing point of the metallic filaments. The analogous crossing of a metallic filament by a pair of close metallic filaments forms a double-quantum dot (DQD). The latter can serve as a two-level qubit cell. Such qubits can be controlled by potentials of three independent metallic filaments inducing the above-mentioned DQD. Besides this "outside" metallic wire control, the DQDs can be connected to each other across the "inside" quantum wires, which have formed these DQDs by crossing. (C) 2005 American Institute of Physics. [DOI: 10.1063/1.1849827]
\end{abstract}

\section{INTRODUCTION}

It is well known that a plane metallic $(M)$ electrode, which is positively charged and separated from a surface of a semiconductor $(S)$ by an isolating barrier $(B)$, attracts electrons by pulling them out of the semiconductor depths and inducing an electron accumulation layer in the semiconductor, which is closely adjacent to the $B / S$ interface [Fig. 1(a)]. The fabricated $n^{+}$contacts that are remote from the considered area of the $B / S$ interface could serve as electron sources, and a necessary positive potential on the electrode $M$ should be set with respect to these contacts. An electric field directed normal to the $B / S$ interface (along the $Z$ axis) together with a heterostructural potential of the $B / S$ interface forms a quasitriangular potential well that localizes electron motion in the $Z$ direction. As a result of such localization, this motion is quantized and discrete energy quantization levels appear. An electron motion in directions along the $B / S$ interface (that is, in one of the $X Y$ planes) is not restricted at all and is described by arbitrary two-dimensional (2D) wave vectors with components $k_{x}, k_{y}$.

Such a picture is valid as long as all the sizes of the electrode $M$ in the $X Y$ plane are large: "unlimited." Limitation of one of these sizes leads to an additional electron motion quantization in the accumulation layer induced in the semiconductor $S$. There are two possible cases. In the first one, the thickness, $z_{0}$, of the isolating barrier $B$ layer is small relative to all of the sizes of the electrode $M$ in the $X Y$ plane.

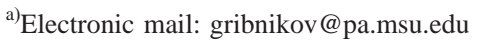

Then we deal with an additional size quantization of a homogeneous electron gas in this plane determined by a configuration and sizes of the electrode $M$. The edge effects appearing near the edges of the electrode $M$ are not substantial for small values of $z_{0}$. The second case, which is really the main subject of our interest in this article, appears if at least one of the electrode's sizes in the $X Y$ plane is smaller than the distance $z_{0}$ between this electrode $M$ and the $B / S$ interface (see Fig. 1). The simplest example of such a situation appears when the filament $M$ size $\left(\sim d_{0}\right)$ is much smaller than $z_{0}$. For simplicity (in order not to increase the quantity of considered materials and interfaces), we have assumed that such a filament is placed into the isolating barrier $B$ medium with a dielectric constant $\varepsilon_{B}$, which is smaller than the dielectric constant, $\varepsilon_{S}$, of the semiconductor $S$. The electron states induced by this filament potential in the semiconductor $S$ (similar to the filament $M$ ) are substantially localized in two directions - along the $Z$ axis (normal to the $B / S$ interface) and along the $X$ axis [normal to the filament, which is parallel to the $B / S$ interface; see Fig. 1(b)]. They are delocalized only along the $Y$ axis, which is parallel to the filament $M$. So, the positively charged filament $M$ induces a quantum wire $(\mathrm{QWr})$ in the semiconductor $S$, directly under the $B / S$ interface. Note that the metallic filament $M$ itself can both be and not be a metallic QWr. The above-mentioned induced quantum wires are considered in Sec. II.

Generally speaking, two identical parallel metallic filaments $M_{1}$ and $M_{2}$, equally distant from the $B / S$ interface [see Fig. 1(c)] do not induce two quantum wires. Being 
(a)

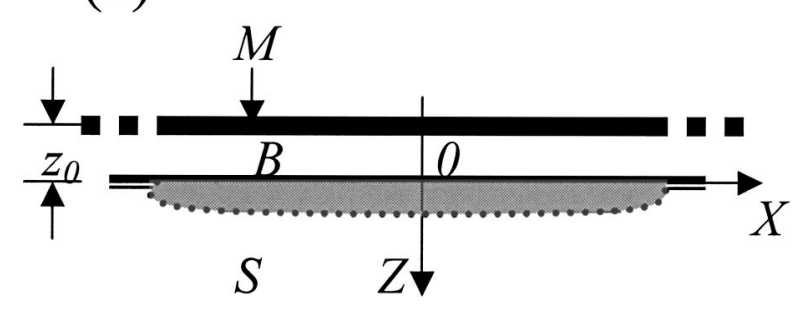

(b)
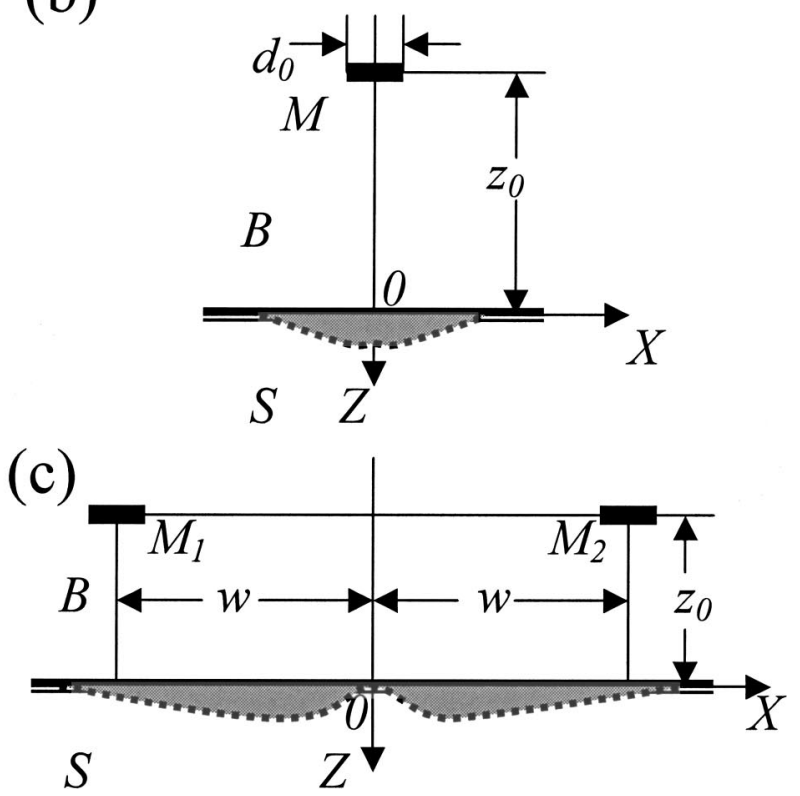

FIG. 1. Induced quantum structures in the semiconductor $S$ under the $B / S$ interface: (a) two-dimensional electron gas (2DEG) induced by the metallic gate $M$; (b) quantum wire (QWr) induced by the metallic filament gate $M$; (c) double-quantum wire (DQWr) induced by the metallic filament gates $M_{1}$ and $M_{2}$.

placed very close to each other, the filaments $M_{1}$ and $M_{2}$ induce a single collective QWr. But if the distance between them, $2 w$, is sufficiently large (i.e., exceeds $2 z_{0}$ ), such a collective QWr splits into two quasiindividual quantum wires, which are connected by tunneling. This tunnel connection becomes very weak with an increase in the distance $2 w$ between the filaments. Due to this weak connection, a ground quantum electron state in the individual QWr splits into a couple of states. The so-called symmetric-antisymmetric (SAS) splitting detaches the asymmetric first excited state from the symmetric ground (principal) state. The value of this splitting depends not only on the distance $2 w$ but also on the electric charges of the filaments. As a result, we obtain the induced double-quantum wire (DQWr). Once, methods of fabrication and properties of the so-called DQWrs were subjects of some pioneering experimental works and numerous theoretical publications. We will return to this topic in Sec. III where we consider the above-presented DQWrs induced by a pair of parallel metallic filaments, which are not connected by tunneling with each other.

In Sec. IV, we switch from parallel metallic filaments to crossed ones. A crossed pair of positively charged filaments $M_{1}$ and $M_{2}$ [Fig. 2(a)] is able to localize an electron under (a)

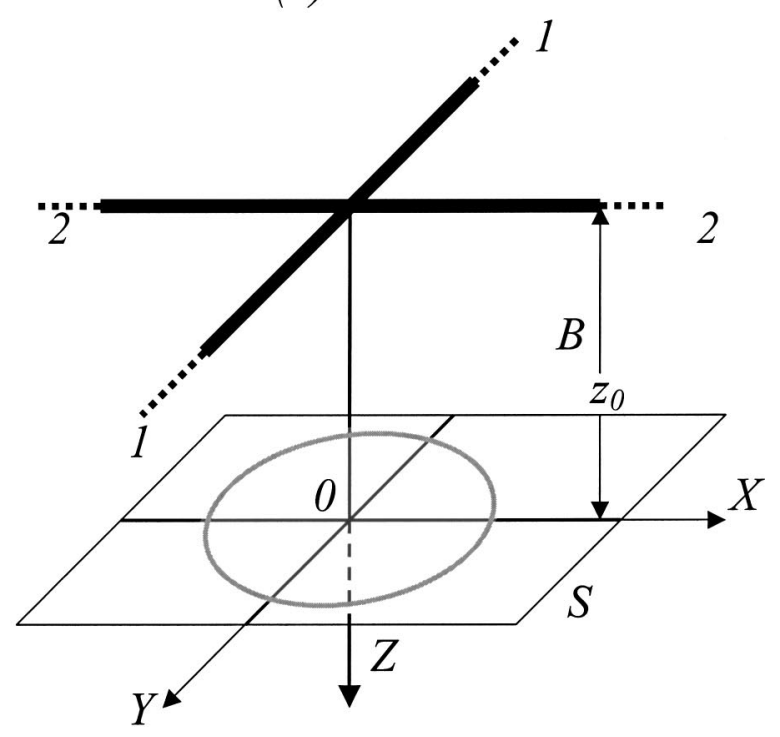

(b)

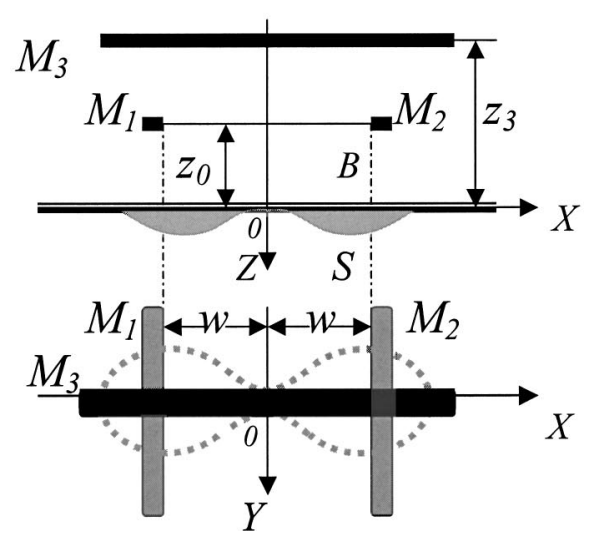

FIG. 2. (a) Quantum dot (QD) induced under the $B / S$ interface by the charged metallic filaments 1-1 and 2-2 crossed with each other in the same plane. (b) Double-quantum dot (DQD) induced under the $B / S$ interface as a result of intersection of the pair of charged metallic filaments $M_{1}$ and $M_{2}$ placed in the plane $z=z_{0}$ by the third filament $M_{3}$ placed in the plane $z=z_{3}$.

the point of crossing and induce the so-called quantum dot (QD) in the semiconductor $S$. Naturally, a pair of parallel metallic filaments $M_{1}$ and $M_{2}$ crossed by the third filament $M_{3}$ can form a double-quantum dot [DQD; see Fig. 2(b)] with the S-AS split lowest state if the distance between the filaments $M_{1}$ and $M_{2}$ forming this pair is large enough.

When we consider the crossed filaments $M_{1}$ and $M_{2}$, there appears an additional problem related to the distances $z_{1}$ and $z_{2}$, separating these filaments from the $B / S$ interface. Since such distances and also charges of crossed filaments can be different, we have an additional possibility to substantially enhance the electron spectra of the induced ordinary and double-quantum dots (see Sec. V). Such a possibility of using different levels of disposition of metallic filaments over the $B / S$ interface allows one to produce a DQWr on the basis of two parallel metallic filaments distant from each other only vertically (one over another) and charged differently; a higher one is charged positively and a lower one is charged negatively. This picture is also described in Sec. V. The DQDs obtained on the basis of such a "vertically de- 
signed" DQWr crossed by the third filament (and its QWr) result in a wider range of controlled parameters in comparison with an "ordinary" induced DQD obtained on the basis of a "horizontally designed" DQWr. The controllability can be still higher if instead of the "vertically induced" DQWr we use the still more complicated DQWr induced by a combined system of three metallic filaments. Such a complicated DQWr is also described in Sec. V.

The induced DQDs, which are de facto the filamentgate-potential-controlled two-level electron structures, can be applied as qubit cells in the quantum-information systems. Besides the ordinary and double QDs and QWrs, quantum point contacts (QPCs) are also important elements of these systems. Like the induced QDs, the induced QPCs can be obtained on the basis of two crossed charged metallic filaments, but charges of these filaments should be of the opposite sign. Unlike a QD, which is the closed quantum structure, an induced QPC is an open structure and requires an additional theoretical description outside this article. But in Sec. VI together with consideration of the possible material systems, we consider the simplest setup of the ballistic fieldeffect transistors with metallic filament gates, allowing one to induce and control both QWr channels and QPCs.

\section{INDUCED QUANTUM WIRE}

Let us consider again the interface of the two materials shown in Fig. 1(b): a semiconductor $(S)$ and a barrier $(B)$ having dielectric constants of $\varepsilon_{S}$ and $\varepsilon_{B}<\varepsilon_{S}$, respectively, and also a metallic filament $M$ with a very small transverse size $d_{0}$. This filament is embedded in the barrier medium $B$ and separated from the $B / S$ interface by the distance $z_{0}$. We assume that a small-width filament $M$ has a long but limited length of $-a \leqslant y \leqslant a$, where $a \gg z_{0} \gg d_{0}$. The filament $M$ is assumed to be positively charged and to have a potential $V_{0}$ relative to a very distant sphere of radius $r \gg a$. In a homogeneous medium with a dielectric constant $\varepsilon_{B}$, a potential $V$ around such a wire is given by ${ }^{1}$

$$
V=\left(\Phi_{0} / \varepsilon_{B}\right) \log [(\xi+1) /(\xi-1)],
$$

where $\xi=\left(r_{1}+r_{2}\right) / 2 a, r_{1,2}=\sqrt{x^{2}+z^{2}+(y \pm a)^{2}}$, the origins for $x$ and $z$ are in the center of the wire, and the origin for $y$ is in the middle of the filament. The constant $\Phi_{0}$ with its potential dimension is not a real potential; it is finite if $d_{0} \rightarrow 0$, whereas a realistic potential diverges logarithmically. Not far from the middle of the filament where $x, y, z \ll a$, we have $\xi \cong 1$ $+r^{2} / 2 a^{2}$ with $r=\sqrt{x^{2}+z^{2}}$, and

$$
V \cong\left(\Phi_{0} / \varepsilon_{B}\right) \log \left(1+4 a^{2} / r^{2}\right) \cong\left(2 \Phi_{0} / \varepsilon_{B}\right) \log (2 a / r) .
$$

For long distances from the filament when $R=\left(x^{2}+y^{2}\right.$ $\left.+z^{2}\right)^{1 / 2} \gg a$, we have

$$
V \cong 2 \Phi_{0} a / \varepsilon_{B} R,
$$

that is, the charged piece of the filament, with the length $2 a$, has a full charge of $Q=2 a \Phi_{0}$, and $\Phi_{0}$ is an average charge density in the wire. For the excess electron density of $\sim 10^{5}-10^{6} \mathrm{e} / \mathrm{cm}$, we have $\Phi_{0} \cong 5 \times 10^{-5}-5$ $\times 10^{-4} \mathrm{~g}^{1 / 2} \mathrm{~cm}^{1 / 2} \mathrm{~s}^{-1}=0.015-0.15 \mathrm{~V}$. [In reality, we are interested in still larger values of $\left.\Phi_{0}(\sim 1 \mathrm{~V})\right]$. Of course, a finite filament cannot be charged homogeneously; its edges are charged stronger than its middle part, but an effect of such an inhomogeneity is not substantial if the length $2 a$ is not small. To obtain a potential $V$ in the medium $S$ [see Fig. 1(b)] with a dielectric constant of $\varepsilon_{S} \neq \varepsilon_{B}$, we should change ${ }^{2,3} 1 / \varepsilon_{B}$ by $2 /\left(\varepsilon_{B}+\varepsilon_{S}\right) \equiv 2 / \varepsilon_{\Sigma}$ in Eqs. (1)-(3). In particular, we have instead of Eq. (2)

$$
V \cong\left(4 \Phi_{0} / \varepsilon_{\Sigma}\right) \log (2 a / r),
$$

where $r$ can be written [after taking into account the shifting of the coordinate origin to the $B / S$ interface; see Fig. 1(b)] in the form of $r=\left(x^{2}+z_{0}^{2}+z^{2}+2 z z_{0}\right)^{1 / 2}$. Let us remember that we have assumed $z_{0} \gg d_{0}$.

The potential $V$ given by Eq. (4) can be written in the neighborhood of the line $x=z=0$ in the form

$$
\begin{aligned}
V \cong V_{W}= & \left(4 \Phi_{0} / \varepsilon_{\Sigma}\right)\left[\log \left(2 a / z_{0}\right)-\left(z / z_{0}\right)-\left(x^{2} / 2 z_{0}^{2}\right)\right. \\
& \left.+\left(z^{2} / 2 z_{0}^{2}\right)\right],
\end{aligned}
$$

that is, this potential can be separated in the variables $x$ and $z$ if $z \ll z_{0}$ and $x^{2} \ll z_{0}^{2}$. Below we will make sure that these inequalities are difficult to satisfy for reasonable values of $z_{0}$ and $\Phi_{0}$. Therefore, the separability of the variables obtainable for the potential in the form (5) can be justified only for the lowest-energy states. The higher states form an infinite system of levels coming closer together with an increase in energy (without the above-mentioned separability in the variables $x$ and $z$ ). If the $B / S$ interface is characterized by a very high heterobarrier, which does not allow electrons to penetrate in the medium of the barrier $B$ from the semiconductor $S$, a boundary condition for a single-electron wave function in this interface is

$$
\Psi(x, y, z=0)=0 .
$$

Besides the potential $V_{W}(x, z)$ [see Eq. (5)], an electron can experience an action of image forces appearing as a result of the different values of $\varepsilon_{S}$ and $\varepsilon_{B}<\varepsilon_{S}$. A potential of the image forces in our case is one dimensional and can be written in the form ${ }^{3}$

$$
V_{I}=e\left(\varepsilon_{S}-\varepsilon_{B}\right) / 2 \varepsilon_{S} \varepsilon_{\Sigma} z
$$

Finally, it is possible that there exists a homogeneous electric field $\Xi_{0}$ induced by charged impurities either in the barrier $B$, or in the $B / S$ interface, or by a remote homogeneously charged electrode. The corresponding potential is

$$
V_{F}=-\Xi_{0} z
$$

The field $\Xi_{0}$ can be both parallel and antiparallel to the $Z$ component of the electric field of the metallic filament,

$$
\Xi_{1}=4 \Phi_{0} / \varepsilon_{\Sigma} z_{0},
$$

presented in Eq. (5). But remember that Eq. (5) is justified only for $z \ll z_{0}$ and $x^{2} \ll z_{0}^{2}$. By summarizing the potentials $V_{W}(x, z), V_{I}(z)$, and $V_{F}(z)$, we obtain

$$
e V(x, z)=e V_{0}+\left(m \omega^{2} / 2\right)\left(z^{2}-x^{2}\right)+e q / z-e \Xi z,
$$

where $\quad V_{0}=\left(4 \Phi_{0} / \varepsilon_{\Sigma}\right) \log \left(2 a / z_{0}\right), \quad \Xi=\Xi_{1}+\Xi_{0}, \quad q=e\left(\varepsilon_{S}\right.$ $\left.-\varepsilon_{B}\right) / 2 \varepsilon_{S} \varepsilon_{\Sigma}, \omega^{2}=4 e \Phi_{0} / m \varepsilon_{\Sigma} z_{0}^{2}$, and $m$ is an electron effective mass for the $X Y$ plane in the semiconductor $S$. Solving the 
three-dimensional (3D)-Schrödinger equation with the potential energy of $-e V(x, z)$ [see Eq. (10)], we obtain for the electron energy

$$
E=E\left(n_{1}, n_{2}, k_{y}\right)=-e V_{0}+\hbar^{2} k_{y}^{2} / 2 m+\hbar \omega\left(n_{1}+1 / 2\right)+E_{n_{2}},
$$

where $k_{y}$ is the wave vector of a "free" motion alongside the $Y$ axis, $n_{1}$ is the quantum number, which describes electron oscillations alongside the $X$ axis, and $E_{n_{2}}$ are the discrete energy levels of the quantization alongside the $Z$ axis in the field with the potential

$$
V_{Z}(z)=-\Xi z+q / z+m \omega^{2} z^{2} / 2 e .
$$

Note that as a result of assuming a finite length, $2 a$, of the metallic filament we should deal with a discrete set of the wave numbers $k_{y}$, but such discreteness is not substantial for large values of $2 a$. A motion in the field of the potential $V_{Z}(z)$ is defined by the wave function $Z\left(n_{2}, z\right)$ that should be found with the boundary conditions of $Z(0)=Z(+\infty)=0$. This means that the last component on the right-hand side of Eq. (12) can be considered only as a small correction. Below, we neglect for our evaluations not only this component but also the image forces component and calculate $E_{Z}\left(n_{2}\right)$ for a triangular quantum well. The wave function $Z\left(n_{2}, z\right)$ in such a potential is described by the formula

$$
Z\left(n_{2}, z\right)=A\left(n_{2}\right) \operatorname{Ai}\left(Z-Z_{n_{2}}\right),
$$

where $\operatorname{Ai}(Z)$ is an Airy function, ${ }^{4} \quad Z=z / z_{C}, \quad z_{C}$ $=\left(\hbar^{2} / 2 M e \Xi\right)^{1 / 3}, A\left(n_{2}\right)$ is a normalizing coefficient, $M$ is an electron effective mass for the $Z$-axis direction, and positive numbers $Z_{n_{2}}$ are the roots of the equation

$$
\operatorname{Ai}\left(-Z_{n_{2}}\right)=0
$$

obtained from the boundary condition $Z\left(n_{2}, 0\right)=0$. Let us write out several of the lowest values ${ }^{4}$ of $Z_{n_{2}}: Z_{1}=2.3381$, $Z_{2}=4.0879, Z_{3}=5.5206, Z_{4}=6.7867$, and so on. We see that the numbers $Z_{n_{2}}$ and the energy levels

$$
E_{n_{2}}=Z_{n_{2}}\left(\hbar e^{\Xi} \Xi\right)^{2 / 3} /(2 M)^{1 / 3}
$$

form the consequence converging with an increase in a number $n_{2}$. The effective mass $M$ can be distinguished from the effective mass $m$ for the motion alongside the $X Y$ plane. Such a situation takes place in the case where the $S$ material is $\mathrm{Si}$, and the $X Y$ plane is one of the $\langle 100\rangle$ planes. Quantization in the $Z$ direction selects only such pair of three pairs of the Si electron valleys, for which really $M>m$.

Let us compare the energy parameter $\delta_{Z}$ $=\left(8 e \hbar \Phi_{0} / z_{0} \varepsilon_{\Sigma}\right)^{2 / 3} / M^{1 / 3}$ describing approximately the energy distance between the $Z$ quantization levels (for $\Xi_{0}=0$ ) with the analogous parameter

$$
\delta_{X}=\hbar \omega=\left(4 e \Phi_{0} / m \varepsilon_{\Sigma}\right)^{1 / 2}\left(\hbar / z_{0}\right)
$$

introduced for the $X$ quantization levels. Their relation $\delta_{Z} / \delta_{X}=2\left(e \Phi_{0} m / \varepsilon_{\Sigma}\right)^{1 / 6}\left(z_{0} m / \hbar M\right)^{1 / 3}$ for $z_{0}=10^{-6} \mathrm{~cm}, \varepsilon_{\Sigma}=\varepsilon_{B}$ $+\varepsilon_{S}=15$, and $\Phi_{0}=0.015 / 0.15 \mathrm{~V}$ could be varied in the limits of $0.5 / 2.0$. The energy $\delta_{X}$ for these numbers is equal to $\sim(0.5 / 1.5) 10^{-2} \mathrm{eV}$. The sizes of the wave functions in the $Z$ and $X$ directions can be evaluated by the values $\Delta_{Z}$ $=\hbar^{2 / 3}\left(\varepsilon_{\Sigma} z_{0} / e M \Phi_{0}\right)^{1 / 3}$ and $\Delta_{X}=\left(\hbar z_{0}\right)^{1 / 2}\left(\varepsilon_{\Sigma} / e m \Phi_{0}\right)^{1 / 4}$, respectively, and we have

$$
\Delta_{Z} / z_{0}=\left(\hbar / z_{0}\right)^{2 / 3}\left(\varepsilon_{\Sigma} / e M \Phi_{0}\right)^{1 / 3}
$$

and

$$
\Delta_{X}^{2} / 2 z_{0}^{2}=\left(\hbar / 2 z_{0}\right)\left(\varepsilon_{\Sigma} / e m \Phi_{0}\right)^{1 / 2} .
$$

The approximation used in Eq. (5) is valid if both $\Delta_{Z} / z_{0}$ $\ll 1$ and $\Delta_{X}^{2} / 2 z_{0}^{2} \ll 1$. In the Si case, the second of these strong inequalities is more critical. This inequality can be satisfied for $z_{0}=10^{-6} \mathrm{~cm}, m=10^{-28} \mathrm{~g}$, and $\varepsilon_{\Sigma}=\varepsilon_{B}+\varepsilon_{S}=15$ if $\Phi_{0}$ $\geqslant 0.15 \mathrm{~V}$ and for $z_{0}=3 \times 10^{-6} \mathrm{~cm}$ if $\Phi_{0} \geqslant 0.015 \mathrm{~V}$. Note that insufficient fulfillment of the above-mentioned strong inequalities does not lead to missing qualitative results but our approximate analytic evaluations become insufficiently accurate.

We have shown above that a QWr could be "grown" directly under the $B / S$ interface, over which a thin metallic filament is really grown. Parameters of the QWr are controlled by the parameters and an electric charge (a potential!) of the filament. The quasiequidistant level system of the $X$ quantization described by the numbers $n_{1}$ is combined in this QWr with levels of the $Z$ quantization described by the numbers $n_{2}$. The above-mentioned image forces, which are not connected with the filament, force electrons out of the interface and decrease energy distances between the $Z$ quantization levels. The homogeneous electric field $\Xi_{0}$, which is parallel to the field $\Xi_{1}$ under the filament, increases these distances.

\section{INDUCED DOUBLE-QUANTUM WIRE}

In the case of the same two parallel finite $(|y| \leqslant a)$ metallic filaments placed over the $B / S$ interface in the same distance $z_{0}$ and in the distance $2 w$ from each other [Fig. 1(c)], the electric potential in the semiconductor $S$ could be presented by the formula

$$
V(x, y, z)=\left(2 \Phi_{0} / \varepsilon_{\Sigma}\right) \log \left[\left(\xi_{1}+1\right)\left(\xi_{2}+1\right) /\left(\xi_{1}-1\right)\left(\xi_{2}-1\right)\right],
$$

where $\quad \xi_{1,2}=\left\{\left[(x \pm w)^{2}+\left(z+z_{0}\right)^{2}+(y-a)^{2}\right]^{1 / 2}+\left[(x \pm w)^{2}+(z\right.\right.$ $\left.\left.\left.+z_{0}\right)^{2}+(y+a)^{2}\right]^{1 / 2}\right\} / 2 a$. If $x, y, z, w \ll a$, we have

$$
\xi_{1,2} \cong 1+\left[(x \pm w)^{2}+\left(z+z_{0}\right)^{2}\right] / 2 a^{2} .
$$

Of course, Eq. (18) is invalid near the edges $|y| \cong a$ of this filament pair but this is not essential because we are interested in the middle parts of the filaments, which are distant from the edges. Taking into account Eq. (19), Eq. (18) can be rewritten in the form

$$
V=\left(8 \Phi_{0} / \varepsilon_{\Sigma}\right)\left\{\log \left[2 a /\left(z_{0}^{2}+w^{2}\right)^{1 / 2}\right]-(1 / 4) \log F(x, z)\right\},
$$

where

$$
F(x, z)=\left\{4 w^{2}\left(z+z_{0}\right)^{2}+\left[x^{2}-w^{2}+\left(z+z_{0}\right)^{2}\right]^{2}\right\} /\left(w^{2}+z_{0}^{2}\right)^{2},
$$

and $z \geqslant 0$. At the $B / S$ interface, where $z=0$, the function $F(x, z)$ transfers into 


$$
F(x, 0)=\left[4 w^{2} z_{0}^{2}+\left(x^{2}-w^{2}+z_{0}^{2}\right)^{2}\right] /\left(w^{2}+z_{0}^{2}\right)^{2} .
$$

The function $F(x, 0)$ is increasing monotonically with an increase in $x^{2}$ if $w^{2} \leqslant z_{0}^{2}$ and experiences a maximum at $x=0$ and minimums at $x^{2}=x_{m}^{2} \equiv w^{2}-z_{0}^{2}$ when $w^{2}>z_{0}^{2}$. In the last case, the electron potential energy $-e V(x, z)$ appearing in the Schrödinger equation contains the analogous maximum and minimums at $z=0$ together with the function $F(x, 0)$. A switch from the single-minimum structure of the potential energy $-e V(x, z)$ at $w^{2} \leqslant z_{0}^{2}$ to the double-minimum structure at $w^{2}>z_{0}^{2}$ leads to the substantial reconstruction of the spectrum of the quantized single-electron states for the QWr induced by the above-mentioned pair of the parallel metallic filaments. This QWr demonstrates a shift from the ordinary single QWr, which is similar to the ones considered in Sec. II, at $w^{2} \leqslant z_{0}^{2}$ to the double QWr (that is DQWr) realized if the inequality $w^{2}>z_{0}^{2}$ is satisfied with a sufficient reserve. The distinctive signs of such a DQWr are contained in the structure of its lower quantized levels: the two lowest levels in the DQWr should be very close to each other. This level couple is formed by the ground symmetric electron state and the first excited antisymmetric state. The interspace between the levels is determined by the tunnel symmetricantisymmetric (S-AS) splitting of the ground state of the conditional half of the considered DQWr. It decreases exponentially with an increase in the height and the width of the potential barrier separating the potential energy minimums. The interminimum distance is equal to $2 x_{m}=2\left(w^{2}-z_{0}^{2}\right)^{1 / 2}$ and determined by the metallic filaments' positions only. The barrier height, which is equal to

$$
\begin{aligned}
e \delta V & =\left|V(0,0)-V\left(x_{m}, 0\right)\right| \\
& =\left(4 e \Phi_{0} / \varepsilon_{\Sigma}\right)\left|\log \left[1-\left(w-z_{0}\right)^{2} /\left(w^{2}+z_{0}^{2}\right)\right]\right| \\
& \cong\left(2 e \Phi_{0} / \varepsilon_{\Sigma}\right)\left(w-z_{0}\right)^{2} / z_{0}^{2}
\end{aligned}
$$

if $w^{2}-z_{0}^{2} \ll 2 z_{0}^{2}$, depends also on the charge of the filaments, $\Phi_{0}$. This height should exceed (and with a sufficiently large reserve) the energy, $\hbar \omega_{m} / 2$, of the conditional quantization for the ground electron state in the energy minimum at $x$ $= \pm x_{m}$. The latter can be evaluated in the form: $\hbar \omega_{m} / 2$ $=(\hbar \omega / 2) 4 z_{0}^{2}\left(w^{2}-z_{0}^{2}\right) /\left(w^{2}+z_{0}^{2}\right)^{2} \cong(\hbar \omega / 2)\left(w^{2}-z_{0}^{2}\right) / z_{0}^{2} \cong \hbar \omega(w$ $\left.-z_{0}\right) / z_{0}$, where $\omega^{2}=4 e \Phi_{0} / m \varepsilon_{\Sigma} z_{0}^{2}$, as in Eq. (10). Then the condition $e \delta V>\hbar \omega_{m} / 2$ appears as

$$
\left(e \Phi_{0} m / \varepsilon_{\Sigma}\right)^{1 / 2}\left(w-z_{0}\right)>\hbar
$$

or

$$
\lambda \equiv \hbar\left(\varepsilon_{\Sigma} / e \Phi_{0} m\right)^{1 / 2}<w-z_{0} .
$$

Remember that we consider in Eqs. (22), (23), and (23a) only a small preponderance of $w$ over $z_{0}: w-z_{0} \ll z_{0}$. This requirement is compatible with Eqs. (23) and (23a) if

$$
2 z_{0} \gg \lambda \text {. }
$$

Equation (24) accurately coincides with the condition $\Delta_{X}^{2} / 2 z_{0}^{2} \ll 1$ [see Eq. (17b)] considered in Sec. II, which has substantiated the simplified formulas of the $X$ quantization for the induced ordinary QWr and allowed us to obtain in that case the ground-state level with the energy $\hbar \omega / 2$. In the case considered here, Eq. (24) jointly with Eq. (23) allows us

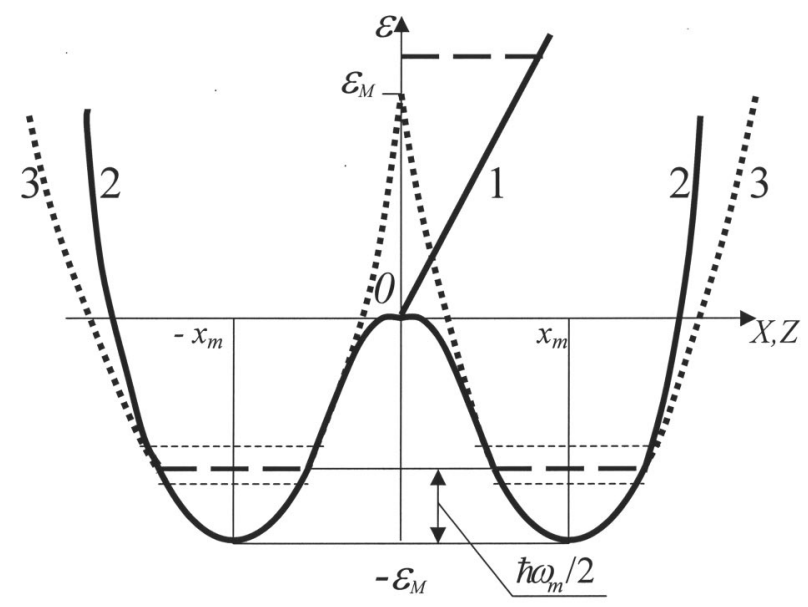

FIG. 3. Potential energy $e \delta V(x, z)$ [see Eq. (25)] of the electron in the DQWr induced by the pair of charged filaments $M_{1,2}$, in the case when $\lambda$ $\ll w-z_{0} \ll z_{0}$. The straight line 1 corresponds to the component $e \delta V(0, z)$ and the solid line 2 corresponds to the component $e \delta V(x, 0)$. The line 3 corresponds to the approximate parabolas $e \delta V(x, 0) \cong \varepsilon_{M}\left[-1+2\left(x \mp x_{m}\right)^{2} / x_{m}^{2}\right]$, where $\left.\varepsilon_{M}=\left(2 e \Phi_{0} / \varepsilon_{\Sigma}\right)\left[\left(w^{2}-z_{0}^{2}\right) /\left(w^{2}+z_{0}^{2}\right)\right]^{2} \cong\left(2 e \Phi_{0} / \varepsilon_{\Sigma}\right)\left(w-z_{0}\right)^{2} / z_{0}^{2}\right)$. These parabolas lead to the equidistant quantization levels $\varepsilon_{n}=\hbar \omega_{m}(n+1 / 2)$.

to obtain only the much shallower S-AS split level with the energy $\hbar \omega_{m} / 2$. Deepening of this level requires an increase in the charges, $\Phi_{0}$, of the filaments.

The S-AS splitting of the lowest level in the abovedescribed DQWr is determined by the potential energy obtained from Eq. (20)

$$
\begin{aligned}
e \delta V(x, z)= & \left(2 e \Phi_{0} / \varepsilon_{\Sigma}\right) \log F(x, z) \\
\cong & \left(2 e \Phi_{0} / \varepsilon_{\Sigma}\right)\left[-2\left(w^{2}-z_{0}^{2}\right) x^{2} /\left(w^{2}+z_{0}^{2}\right)^{2}\right. \\
& \left.+x^{4} /\left(w^{2}+z_{0}^{2}\right)^{2}+4 z z_{0} /\left(w^{2}+z_{0}^{2}\right)\right] .
\end{aligned}
$$

Formula (25) is justified when each of the components in the brackets is small in comparison to (1). We do not keep in these brackets the small component $4 z z_{0} x^{2} /\left(w^{2}+z_{0}^{2}\right)^{2}$, which hinders separation of the variables, and assume that this component is a higher-order infinitesimal and can be neglected. The potential energy e $\delta V(x, z)$ in Eq. (25) describes an aggregate of the one-dimensional (1D)-(QW) quantum well along the $X$ axis and the triangular 1D-QW along the $Z$ axis (see Fig. 3). The desired S-AS splitting of the principal and the first excited states of the DQWr can be presented by the approximate formula ${ }^{5}$

$$
E_{\mathrm{AS}}-E_{\mathrm{S}}=\left(h \omega_{m} / 2 \pi\right) \exp \left[-(2 / \hbar) \int_{0}^{a}|p| d x\right],
$$

where the quasiclassic momentum $p(x)$ can be written in the form

$$
\begin{aligned}
p(x)= & \left\{2 m \left[\left(2 e \Phi_{0} / \varepsilon_{\Sigma}\right)\left(w^{2}-z_{0}^{2}-x^{2}\right)^{2} /\left(w^{2}+z_{0}^{2}\right)\right.\right. \\
& \left.\left.-\hbar \omega_{m} / 2\right]\right\}^{1 / 2} \\
\cong & \left(2 \hbar / \lambda z_{0}\right)\left[\left(w-z_{0}-x^{2} / 2 z_{0}\right)^{2}-\lambda\left(w-z_{0}\right)\right]^{1 / 2}
\end{aligned}
$$

and the top limit of the integral in Eq. (26a) is $a=x_{m}$ $-\left(\lambda z_{0}\right)^{1 / 2} \cong z_{0}^{1 / 2}\left[2^{1 / 2}\left(w-z_{0}\right)^{1 / 2}-\lambda^{1 / 2}\right]$. When the inequality in Eq. (23a) is strong, the exponential index in Eq. (26) is equal to $\left[(8 \sqrt{2})\left(w-z_{0}\right)^{3 / 2} / 3 \lambda z_{0}^{1 / 2}\right]$, and the S-AS splitting is small if 


$$
\pi \exp \left[(8 \sqrt{2})\left(w-z_{0}\right)^{3 / 2} / 3 \lambda z_{0}^{1 / 2}\right] \gg 1 .
$$

As in the case of the inequality in Eq. (23a), we need small values of the parameter $\lambda$ and sufficiently large values of $w-z_{0}$. The same tendency takes place if the difference $w$ $-z_{0}$ does not become small in comparison to $z_{0}$.

\section{CROSSED FILAMENTS: AN INDUCED QUANTUM DOT}

Let us turn from the case of two parallel metallic filaments to the case of two crossed filaments. Our interest in the case of crossed filaments has been prompted by the possibility of forming a QD with completely localized electron states. It is known ${ }^{6}$ that an intersection of QWrs generates bound quantum states localized around this intersection. [Generally speaking, formation of such states does not require the "full" crossing (of the +-type or $X$-type). It is sufficient ${ }^{6}$ to create $T$-like or $Y$-like intersections, and even simple angles $(\Gamma$-like $)]$. There have been ${ }^{7,8}$ some experimental versions of crossing QWr on the basis of laterally patterned modulation-doped GaAs/AlGaAs heterostructures fabricated by the use of electron-beam lithography and ionbeam-assisted etching. Unfortunately, the above-mentioned localized states have not been considered in these works. But, on the other hand, such states have been investigated in Refs. 9-13, in which QDs have been obtained as a result of the intersection of the two $T$-shape QWrs. These $T$-QWrs have been manufactured by cleaved edge overgrowth (CEO; see the detailed review and numerous references in Ref. 14). The QDs have been grown by the twofold CEO procedure suggested in Ref. 15 and implemented in Refs. 9-13.

Let us consider two filaments, which are placed perpendicularly to each other and form an equal-arm cross in the plane $z=-z_{0}$ parallel to the $B / S$ interface [see Fig. 2(a)]. In analyzing this case we intend to use Eq. (18) the same as before with some redefinition of functions $\xi_{1}$ and $\xi_{2}$ on the right-hand side. Now we have $\xi_{1,2}=\left(r_{1}^{(1,2)}+r_{2}^{(1,2)}\right) / 2 a_{1,2}$, where $r_{1,2}^{(1)}=\left[x^{2}+\left(z+z_{0}\right)^{2}+\left(y \pm a_{1}\right)^{2}\right]^{1 / 2}$ and $r_{1,2}^{(2)}=\left[\left(x \pm a_{2}\right)^{2}+(z\right.$ $\left.\left.+z_{0}\right)^{2}+y^{2}\right]^{1 / 2} ; a_{1}$ and $a_{2}$ are the lengths of these filaments. Of course, such an approach is very simplified since we assume, in fact, that charge densities in both crossing filaments are constant in the neighborhood of the intersection point that is incorrect. We assume that the uncounted charge redistribution should not be noticeable in the case of very small transverse sizes of the filaments, and the qualitative picture is adequate.

In the neighborhood of the coordinate origin where $x, y$, $z, z_{0} \ll a_{1,2}$ we obtain

$$
\xi_{1,2} \cong 1+(1 / 2)\left(r^{(1,2)} / a_{1,2}\right)^{2}
$$

with $r^{(1)}=\left(x^{2}+z^{2}\right)^{1 / 2}$ and $r^{(2)}=\left(y^{2}+z^{2}\right)^{1 / 2}$. As a result of these redefinitions, we obtain from Eq. (18)

$$
\begin{aligned}
V(x, y, z)= & \left(8 \Phi_{0} / \varepsilon_{\Sigma}\right)\left\{\log \left[2\left(a_{1} a_{2}\right)^{1 / 2} / z_{0}\right]-(1 / 4) \log \left[\left(x / z_{0}\right)^{2}\right.\right. \\
& \left.\left.+\left(1+z / z_{0}\right)^{2}\right]-(1 / 4) \log \left[\left(y / z_{0}\right)^{2}+\left(1+z / z_{0}\right)^{2}\right]\right\} .
\end{aligned}
$$

If $z / z_{0} \ll 1$ and $\left(x / z_{0}\right)^{2},\left(y / z_{0}\right)^{2} \ll 1$, Eq. (29) becomes similar to Eq. (5) describing the ordinary QWr as

$$
\begin{aligned}
V \cong & V_{C}=\left(8 \Phi_{0} / \varepsilon_{\Sigma}\right)\left\{\log \left[2\left(a_{1} a_{2}\right)^{1 / 2} / z_{0}\right]-\left(z / z_{0}\right)\right. \\
& \left.-\left(x^{2}+y^{2}-2 z^{2}\right) / 4 z_{0}^{2}\right\} .
\end{aligned}
$$

But in contrast to the potential $V_{W}$ described by Eq. (5), which localizes the motion in the $X Y$ plane only alongside the $X$ axis, the potential $V_{C}$ localizes the motion in this plane completely. Therefore, the subject described by this potential is a quantum dot (QD). This QD is induced by the crossed charged filament shown in Fig. 2(a). The potential $V_{C}$ presented by Eq. (30) is axially symmetric. But with an increase in $x$ and $y$ (in comparison with $z_{0}$ ) such an axial symmetry gradually disappears. Equipotential lines, which are circles for small values of $\left(x^{2}+y^{2}\right) / z_{0}^{2}<1$, become more and more crosslike with an increase in this value going deep into the channels of the vanishing quantum wires. The energy quantization levels corresponding to the potential $V(x, y, z)=V_{C}$ $+V_{I}+V_{F}$ [see Eqs. (7) and (8)] are given by the formula

$$
E=E_{n_{1}, n_{2}}=-2 e V_{0}^{\prime}+\hbar \omega\left(n_{1}+1\right)+E_{n_{2}}^{\prime},
$$

where $V_{0}^{\prime}=\left(4 \Phi_{0} / \varepsilon_{\Sigma}\right) \log \left[2\left(a_{1} a_{2}\right)^{1 / 2} / z_{0}\right]$ and the frequency $\omega$ is the same as in Eqs. (10) and (11). The coefficient 2 before $e V_{0}^{\prime}$ is connected with double numbers of the filaments with the same charge density $\Phi_{0}$ in the same place. As a result of the same reason, we should replace the expression for $V_{Z}(z)$ in Eq. (12) by the new expression $V_{Z}^{\prime}(z)=-\left(2 \Xi_{1}+\Xi_{0}\right) z$ $+q / z+m \omega^{2} z^{2} / e$ in order to calculate $E_{n_{2}}^{\prime}$ in Eq. (31). The energy of the ground state of the quantization in the $X Y$ plane has also doubled; we have $\hbar \omega$ in Eq. (31) instead of $\hbar \omega / 2$ in Eq. (11), though the distance $\hbar \omega$ between the quantization levels remains the same in the accepted equidistant approximation. But let us note that all such levels in Eq. (11) are nondegenerate and all of them in Eq. (31) are degenerate, excluding the level $n_{1}=0$. The degeneration multiplicity is equal to $n_{1}+1$, that is, the first excited state is double degenerated, etc. Since for large numbers of $n_{1}$ the approximate potential, which is proportional to $\left(x^{2}+y\right)^{2} / 4 z^{2}$ in Eq. (30), is insufficiently accurate, these levels become nonequidistant and split into nondegenerate (or less degenerate) levels. As a result, the structure of quantization levels for our QD could be substantially enriched.

Up to now, we have assumed that the considered intersected filaments forming the QD are the same. They have the same cross-section shape and size, the same charge density $\Phi_{0}$, and the same distance $z_{0}$ from the $B / S$ interface. But all these conditions are not essential in order to form the QD. The induced QWrs and QDs in the semiconductor $S$ are formed directly under the $B / S$ interface independently of distances between charged filaments (or any other charged sources) and this $B / S$ interface. These filaments can be the same or different. They can be short circuited to each other and have the same potential, but they can also be isolated from each other and have different potentials. Depending on these potentials and on real shapes and sizes of these filaments (which could be not only filaments), we can create different potential distributions in the semiconductors under the $B / S$ interface and form various induced structures including dots, wires, potential barriers, vast electron reservoirs, and contact systems. 


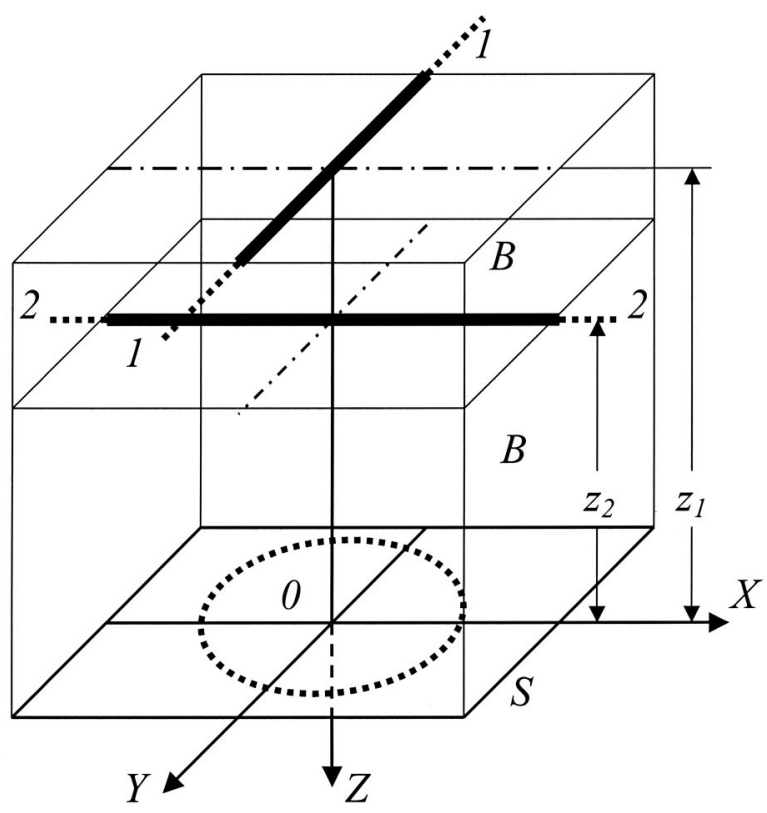

FIG. 4. Quantum dot (QD) induced under the $B / S$ interface by the crossed charged metallic filaments 1-1 and 2-2 placed in the different planes in parallel to the $B / S$ interface.

Let us consider the analogous to Eq. (29) expression for the potential in the close neighborhood $\left(x, y, z, z_{1,2} \ll a_{1,2}\right)$ of the crossing point of two differently charged filaments, which are parallel to the $B / S$ interface and differently distant from this interface $\left(z_{1,2}\right.$ are the distances from the interface; see Fig. 4). As before, we assume that these filaments are perpendicular to each other and their projections on the interface are crossed in the middle. Instead of the single charge density $\Phi_{0}$, we introduce the two analogous parameters $\Phi_{1}$ and $\Phi_{2}$. As a result of such changes, instead of Eq. (29) we obtain

$$
V=\left(4 / \varepsilon_{\Sigma}\right)\left(\Gamma_{1}+\Gamma_{2}\right)
$$

where $\quad \Gamma_{1,2}=\Phi_{1,2}\left\{\log \left(2 a_{1,2} / z_{1,2}\right)-(1 / 2) \log \left[1+2\left(z / z_{1,2}\right)\right.\right.$ $\left.\left.+\left(r^{(1,2)} / z_{1,2}\right)^{2}\right]\right\}$. The analogous approximate formula can be obtained if we use Eq. (32) instead of Eq. (30); the approximate expressions for $\Gamma_{1,2}: \Gamma_{1}=\Phi_{1}\left[\log \left(2 a_{1} / z_{1}\right)-\left(z / z_{1}\right)+\left(z^{2}\right.\right.$ $\left.\left.-x^{2}\right) / 2 z_{1}^{2}\right]$ and $\Gamma_{2}=\Phi_{2}\left[\log \left(2 a_{2} / z_{2}\right)-\left(z / z_{2}\right)+\left(z^{2}-y^{2}\right) / 2 z_{2}^{2}\right]$. As a result of the inequality $z_{1} \neq z_{2}$, we have lost the symmetry in relation to the transformation $x \rightarrow y, y \rightarrow x$. Therefore, instead of Eq. (31) we have

$E=E_{n_{1}^{\prime}, n_{1}^{\prime \prime}, n_{2}}=-2 e V_{0}^{\prime}+\hbar \omega^{\prime}\left(n_{1}^{\prime}+1 / 2\right)+\hbar \omega^{\prime \prime}\left(n_{1}^{\prime \prime}+1 / 2\right)+E_{n_{2}}^{\prime \prime}$,

where $V_{0}^{\prime}=\left(2 / \varepsilon_{\Sigma}\right)\left[\Phi_{1} \log \left(2 a_{1} / z_{1}\right)+\Phi_{2} \log \left(2 a_{2} / z_{2}\right)\right]$,

$$
\omega^{\prime 2}=4 e \Phi_{1} / m \varepsilon_{\Sigma} z_{1}^{2},
$$

and

$$
\omega^{\prime \prime 2}=4 e \Phi_{2} / m \varepsilon_{\Sigma} z_{2}^{2} .
$$

The energy $E_{n_{2}}^{\prime \prime}$ has the same structure as the energies $E_{n_{2}}$ and $E_{n_{2}}^{\prime}$ in formulas (11) and (31), being different only due to the redefinition of parameters. Formula (33) demonstrates the above-indicated lifting of degeneracy as a result of the axial symmetry disappearing. If $n_{1}^{\prime}=n_{1}^{\prime \prime}=0$, we have the single level of the "zero" electron oscillations with the energy of $E_{0}=\hbar \omega=\hbar\left(\omega^{\prime}+\omega^{\prime \prime}\right) / 2$, as before. But the doubly degenerate level $n_{1}=1$ with the energy of $E_{1}=2 \hbar \omega$ splits into two nondegenerate levels: (1). $n_{1}^{\prime}=1, n_{1}^{\prime \prime}=0, E_{1,0}=\hbar\left(\omega+\omega^{\prime}\right)$ and (2). $n_{1}^{\prime}=0, n_{1}^{\prime \prime}=1, E_{0,1}=\hbar\left(\omega+\omega^{\prime \prime}\right)$. The triply degenerate level $n_{1}$ $=2$ with the energy of $E_{2}=3 \hbar \omega$ splits onto three nondegenerate levels: (1). $n_{1}^{\prime}=2, n_{1}^{\prime \prime}=0, E_{2,0}=\hbar\left(\omega+2 \omega^{\prime}\right),(2) . n_{1}^{\prime}=1$, $n_{1}^{\prime \prime}=1, \quad E_{1,1}=\hbar\left(\omega+\omega^{\prime}+\omega^{\prime \prime}\right), \quad(3) . \quad n_{1}^{\prime}=0, \quad n_{1}^{\prime \prime}=2, \quad E_{0,2}=\hbar(\omega$ $\left.+2 \omega^{\prime \prime}\right)$, etc. Differences between the split energies are small if frequencies $\omega, \omega^{\prime}$, and $\omega^{\prime \prime}$ are close to each other. In the opposite case, the level systematics can be substantially disrupted. Equality or inequality of the above-mentioned frequencies depends on the values of $\Phi_{1} / z_{1}^{2}$ and $\Phi_{2} / z_{2}^{2}$ [see Eqs. (34a) and (34b)] and can be controlled not only by the filament design but also by the filament charges (that is, by their potentials).

\section{DOUBLE-QUANTUM DOTS AND OTHER VERSIONS OF THE DOUBLE-QUANTUM WIRES}

In Sec. III, we have considered a certain method of formation of DQWrs by the fabrication of two parallel metallic filaments placed in the $X Y$ plane, which is parallel to the $B / S$ interface and over it. These filaments should be sufficiently distant from each other (the distance $2 w$ should be somewhat larger than $2 z_{0}$ ). Naturally, the analogous method is also suitable for the formation of DQDs, which can be created by crossing the above-described pair of parallel filaments with the third metallic filament $M_{3}$ [see Fig. 2(b)]. In other words, we should form a pair of not very long-distant QWrs using the method "by filament crossing" described in Sec. IV. We need this "not very long" distance in order not to obtain the S-AS level splitting that is excessively small. The transverse third filament $M_{3}$ is obliged neither to be placed in the same $X Y$ plane with the parallel pair nor to be equally charged with each of them. The potential, creating each of two QDs in the DQD, can be presented in the form [compare with Eq. (30)]

$$
\begin{aligned}
V= & \left(4 / \varepsilon_{\Sigma}\right)\left\{\Phi_{3} \log \left(2 a_{3} / z_{3}\right)+2 \Phi_{0} \log \left[2 a /\left(z_{0}^{2}+w^{2}\right)^{1 / 2}\right]\right. \\
& +\left(\Phi_{0} / 2\right)\left(w^{2}-z_{0}^{2}\right)^{2} /\left(w^{2}+z_{0}^{2}\right)^{2}-z\left[\Phi_{3} / z_{3}\right. \\
& \left.+2 \Phi_{0} z_{0} /\left(w^{2}+z_{0}^{2}\right)\right]-\left(\Phi_{3} / 2\right) \\
& \left.\times\left(y / z_{3}\right)^{2}-\left(\Phi_{0} / 2\right) x^{\prime 2}\left(x_{m}+x\right)^{2} /\left(w^{2}+z_{0}^{2}\right)^{2}\right\}
\end{aligned}
$$

where $x_{1}=x-x_{m}, \Phi_{3}$ is the charge of the metallic filament $M_{3}$ placed at the distance $z_{3}$ from the $B / S$ interface, and $\Phi_{0}$ is the charge of each of the filaments $M_{1,2}$, placed at the distance $z_{0}$ from the $B / S$ interface. If $w-z_{0} \ll 2 z_{0}$, the last two components in the curly brackets on the right-hand side of Eq. (35) can be expressed in the form

$$
-(1 / 2)\left[\Phi_{3}\left(y / z_{3}\right)^{2}+2 \Phi_{0} x^{\prime 2}\left(w-z_{0}\right) / z_{0}^{3}\right] .
$$

In the case of the equal (or close to each other) values of $\Phi_{0}$ and $\Phi_{3}$ and simultaneously also $z_{3}^{2}$ and $z_{0}^{2}$, we could obtain a very anisotropic QD and, as a result, a very distinctive DQD. The standard situation can be approximately restored if $\Phi_{3} / z_{3}^{2} \approx 2 \Phi_{0}\left(w-z_{0}\right) / z_{0}^{3}$. This means that the filament $M_{3}$ should be noticeably distant from the $B / S$ interface in com- 
(a)

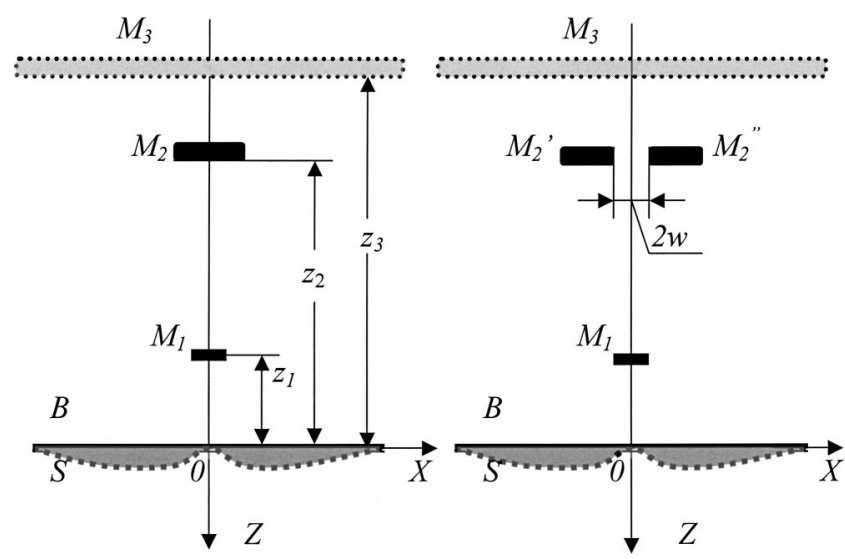

FIG. 5. (a) Double-quantum dot induced by the pair of charged metallic filaments $M_{1}$ and $M_{2}$ placed one over another. The filament $M_{1}$ is negatively charged and the filament $M_{2}$ is positively charged. (b) Double-quantum dot induced by the negatively charged filament $M_{1}$ and the pair of positively charged filaments $M_{2}^{\prime}$ and $M_{2}^{\prime \prime}$. In both cases, the positively charged filaments $M_{3}$ directed along the $X$ axis induce the double-quantum dots under the crossing points.

parison to the filaments $M_{1,2}$ and the charge $\Phi_{3}$ should be small in comparison to the charge $\Phi_{0}$.

Of course, the above-described designs of DQWrs and DQDs are not unique. Below, we consider one more. It includes two parallel metallic filaments $M_{1}$ and $M_{2}$ placed one over the other [see Fig. 5(a)] in the same vertical $Y Z$ plane in the distance $z_{2}-z_{1}$ from one another $\left(z_{1}\right.$ and $z_{2}$ are the distances from the filaments $M_{1}$ and $M_{2}$ to the $B / S$ interface). As before, we assume that the transverse filament sizes of both $M_{1}$ and $M_{2}$ are small (disappearingly small!) in comparison with both $z_{1}$ and $z_{2}$. This means that the charged filaments $M_{1}$ and $M_{2}$ do not screen practically with one another. The potential of these filaments in the semiconductor $S$ is presented by Eq. (32) with the new values of $\Gamma_{1,2}$

$$
\begin{aligned}
\Gamma_{1,2}= & \Phi_{1,2}\left\{\log \left(2 a_{1,2} / z_{1,2}\right)-(1 / 2)\right. \\
& \left.\times \log \left[1+2\left(z / z_{1,2}\right)+r^{2} / z_{1,2}^{2}\right]\right\},
\end{aligned}
$$

where $r^{2}=x^{2}+z^{2}$. As before, we assume that $x, z \ll z_{1}$ and obtain the formula, which is analogous to Eq. (30)

$$
V \cong\left(4 / \varepsilon_{\Sigma}\right)\left(\gamma_{1}+\gamma_{2}\right)
$$

with $\gamma_{1,2}=\Phi_{1,2}\left[\log \left(2 a_{1,2} / z_{1,2}\right)-\left(z / z_{1,2}\right)+\left(z^{2}-x^{2}\right) / 2 z_{1,2}^{2}\right]$.

We assume that (1). $\Phi_{1}<0$, but $\Phi_{2}>0$ as before; (2). $\Phi_{2}>\left|\Phi_{1}\right|$, and this inequality should be sufficiently strong in order to provide the next additional inequalities,

$$
\Phi_{2} \log \left(2 a_{2} / z_{2}\right)>\Phi_{1} \log \left(2 a_{1} / z_{1}\right),
$$

$$
\Phi_{2} / z_{2}>\left|\Phi_{1}\right| / z_{1}
$$

but

$$
\Phi_{2} / z_{2}^{2}<\left|\Phi_{1}\right| / z_{1}^{2},
$$

that is

$$
\frac{\left|\Phi_{1}\right|}{\Phi_{2}}>\frac{z_{1}^{2}}{z_{2}^{2}}>\frac{\Phi_{1}^{2}}{\Phi_{2}^{2}}
$$

The above-written inequalities (38) and (39) mean that the vertical filament pair $M_{1}$ and $M_{2}$ behaves itself as a positively charged filament for sufficiently large distances when $x^{2}, z^{2} \geqslant z_{2}^{2}$ since the realistic positive charge $\Phi_{2}>0$ significantly exceeds the negative charge $\left|\Phi_{1}\right|\left(\Phi_{1}<0\right)$ and dominates everywhere excluding a small neighborhood $x^{2}, z^{2}$ $\leqslant z_{1}^{2}$ of the filament $M_{1}$. The negative charge of the filament $M_{1}$, which dominates in the above-indicated small neighborhood, pushes out an electron from this space and depletes it. In this case, the minimums of the potential energy are reached for $z=0$ at $x= \pm x_{m}$ (see Fig. 3), where

$$
x_{m}^{2}=\left(\left|\Phi_{1}\right| z_{2}^{2}-\Phi_{2} z_{1}^{2}\right) /\left(\Phi_{2}-\left|\Phi_{1}\right|\right) .
$$

If inequalities (39a) take place, the right-hand side of Eq. (40) is positive. The excess of the potential energy $e V(0,0)$ over the energy $e V\left(x_{m}, 0\right)$ can be presented by the formula

$$
\begin{aligned}
\delta= & e V(0,0)-e V\left(x_{m}, 0\right) \\
= & \left(2 e / \varepsilon_{\Sigma}\right)\left\{-\left|\Phi_{1}\right| \log \left(\left|\Phi_{1}\right| z_{2}^{2} / \Phi_{2} z_{1}^{2}\right)+\left(\Phi_{2}-\left|\Phi_{1}\right|\right) \log [(1\right. \\
& \left.\left.\left.-z_{1}^{2} / z_{2}^{2}\right) /\left(1-\left|\Phi_{1}\right| / \Phi_{2}\right)\right]\right\} \cong\left(2 e / \varepsilon_{\Sigma}\right) \\
& \times\left\{-\left|\Phi_{1}\right| \log \left(\left|\Phi_{1}\right| z_{2}^{2} / \Phi_{2} z_{1}^{2}\right)-\left(\Phi_{2}-\left|\Phi_{1}\right|\right)\left[\left(z_{1}^{2} / z_{2}^{2}\right)\right.\right. \\
& \left.\left.-\left|\Phi_{1}\right| / \Phi_{2}\right]\right\} .
\end{aligned}
$$

The third equality in Eq. (41) is justified if $\left|\Phi_{1}\right| \ll \Phi_{2}$ and $z_{1}^{2} \ll z_{2}^{2}$. We also have

$$
\begin{aligned}
e V(x, 0) \cong & e V\left(x_{m}, 0\right) \\
& +\frac{8 e\left(\Phi_{2}-\left|\Phi_{1}\right|\right)^{2}\left[\left(z_{2}^{2} / \Phi_{2}\right)-\left(z_{1}^{2} /\left|\Phi_{1}\right|\right)\right]}{\varepsilon_{\Sigma}\left(z_{2}^{2}-z_{1}^{2}\right)^{2}}\left(x \mp x_{m}\right)^{2}
\end{aligned}
$$

around the positions $x= \pm x_{m}$ of the energy minimums. The frequency $\omega$ of the electron oscillations around $x= \pm x_{m}$ could be found from the formula

$$
\omega^{2}=\left(16 e / m \varepsilon_{\Sigma}\right)\left[\left(\Phi_{2}-\left|\Phi_{1}\right|\right)^{2} /\left(z_{2}^{2}-z_{1}^{2}\right)^{2}\right]\left(z_{2}^{2} / \Phi_{2}-z_{1}^{2} /\left|\Phi_{1}\right|\right) .
$$

The zero oscillation energy $\hbar \omega / 2$ should be noticeably smaller than the height, $\delta$, of the potential barrier separating the above-mentioned minimums

$$
\left(1-z_{1}^{2} / z_{2}^{2}\right)\left|\Phi_{1}\right|\left[\left(e m / \varepsilon_{\Sigma}\right)\left(z_{2}^{2} / \Phi_{2}-z_{1}^{2} /\left|\Phi_{1}\right|\right)^{1 / 2}>\hbar .\right.
$$

Inequality (43) is analogous to Eq. (23) [or (23a)] that is obtained for the DQWrs of the first version. The advantage of the "vertical" second version considered in this section in comparison to the "horizontal" first version is determined by its more varied controllability: parameters of the DQWr depend on two different charges $\Phi_{1}$ and $\Phi_{2}$. By varying these charges, we can transform our DQWr into the ordinary QWr and vary the positions of the energy levels over a very wide range, both in the double version and in the ordinary version. The role of the additional negative-charged filament $M_{1}$ (Fig. 5 ) is analogous to the role of the thin depleting middle gate in the tunnel-barrier split electron waveguides. ${ }^{16}$ The middlegate architecture ${ }^{16-19}$ has been designed for fabrication of DQWrs by tunnel-barrier splitting of an ordinary wide QWr 
in two. An extremely narrow gate, manufactured with the help of the direct-write electron-beam lithography, ${ }^{16,19}$ provides such a splitting. The obtained DQWrs (split electron waveguides) with autonomously contacted halves have been intended for implementation of ballistic resonant tunnel couplers $^{20,21}$ and other resonant devices. Most of such and other devices based on DQWrs and combinations of DQWrs with ordinary QWrs could be designed on the basis of the above-described metallic filaments.

Still more controllability than in the case of the vertically designed DQWrs could be reached for the DQDs designed on the basis of such DQWrs [see Fig. 5(a)]. For transformation of such a DQWr into the DQD we need to introduce the additional filament $M_{3}$, which is perpendicular to the filaments $M_{1}$ and $M_{2}$ and placed at some distance $z_{3}$ from the $B / S$ interface. In Fig. 5 it is placed over the filaments $M_{1}$ and $M_{2}\left(z_{3}>z_{2}>z_{1}\right)$ but it is optional; we only need $z_{2}>z_{1}$ with a certain substantial reserve as before. The elementary theory of such a DQD can be developed by adding the third component $\Gamma_{3}$ to the sum of $\Gamma_{1}+\Gamma_{2}$ on the right-hand side of Eq. (32), where $\Gamma_{1}$ and $\Gamma_{2}$ are presented by Eq. (36). This third component is

$$
\Gamma_{3}=\Phi_{3}\left\{\log \left(2 a_{3} / z_{3}\right)-z / z_{3} \log \left[1+2 z / z_{3}+\left(z^{2}+y^{2}\right) / z_{3}^{2}\right]\right\} .
$$

In addition to the sum of $\gamma_{1}+\gamma_{2}$, this component adds in the right-hand side of Eq. (37) the component

$$
\gamma_{3}=\Phi_{3}\left[\log \left(2 a_{3} / z_{3}\right)-z / z_{3}+\left(z^{2}-y^{2}\right) / 2 z_{3}^{2}\right],
$$

where $\Phi_{3}$ is always positive similar to $\Phi_{2}$. This additional component allows us to replace the inequalities in (38) and (39) by much more liberal ones. Specifically, instead of Eq. (38b) we can use

$$
\Phi_{2} / z_{2}+\Phi_{3} / z_{3}>\left|\Phi_{1}\right| / z_{1}
$$

This inequality in combination with the inequality in (39) leads to

$$
\frac{\left|\Phi_{1}\right|}{\Phi_{2}}>\frac{z_{1}^{2}}{z_{2}^{2}}>\frac{\Phi_{1}^{2}}{\Phi_{2}^{2}}\left(1+\Phi_{3} z_{2} / \Phi_{2} z_{3}\right)^{-2}
$$

instead of Eq. (39a). Such a replacement noticeably widens the controllability area for the considered DQDs.

In particular, let us consider the case when $\left|\Phi_{1}\right|$ is so large that $\left|\Phi_{1}\right| / \Phi_{2}$ exceeds not only $\left(z_{1} / z_{2}\right)^{2}$ but also $z_{1} / z_{2}$. This means that Eq. (38b) together with the right inequality in Eq. (39a) is not fulfilled. As a result of this nonfulfillment, both the DQWr and QWr cannot exist outside the vicinity of the intersection point of the filaments $M_{1}$ and $M_{2}$ by the third filament $M_{3}$, such a $M_{1}+M_{2}$ combination of the filament charges repels electrons from the $B / S$ interface. But the positively charged crossing filament $M_{3}$ changes the situation around the crossing point if the charge $\Phi_{3}$ is sufficiently large, and the inequalities in (46) and (47) are fulfilled. The DQD becomes possible in the neighborhood of the crossing point of the QWr on the basis of the filament $M_{3}$ and the nonexistent (imaginary!) DQWr on the basis of the filaments $M_{1}+M_{2}$. Since fulfillment or nonfulfillment of the inequality in $(38 b)$ is determined by the charge combination of the three participating charged filaments, we can deal with both the "semi-isolated" DQDs strung onto the QWr on the basis of the filament $M_{3}$ and the DQDs also connected with the DQWrs growing alongside the filaments $M_{1}+M_{2}$ (if they exist).

The above-considered DQWrs, on the basis of the two metallic filaments placed in the same vertical plane [Fig. 5(a)], have a very substantial disadvantage besides the above-mentioned advantages. It could be formulated as the instability of the S-AS splitting of the ground quantum states as a result of a small asymmetry of the left and right sides (halves) of the induced DQWr. In the case of the horizontally designed DQWr on the basis of the two independent metallic filaments placed in the approximately same horizontal plane [Fig 1(c)], the small controllable potential difference could compensate for any small asymmetry of the forming filaments $M_{1}$ and $M_{2}$ [such as unequal charges $\left(\Phi_{1} \neq \Phi_{2}\right)$ or unequal distances from the $B / S$ interface $\left(z_{1} \neq z_{2}\right)$, etc.] As a result of such compensation, we obtain the minimum value of the resonant S-AS splitting. But in the case of the vertically designed DQWrs [Fig. 5(a)], we miss such a possibility, and any technological asymmetry (such as incomplete verticality or small asymmetry of the filaments) leads to missing of the desirable minimum S-AS splitting. To reestablish stability, we can exchange the positively charged filament $M_{2}$ in Fig. 5(a) by the pair of independent filaments $M_{2}^{\prime}$ and $M_{2}^{\prime \prime}$ placed at the same distance, $z_{2}^{\prime}=z_{2}^{\prime \prime}$, from the $B / S$ interface [see Fig. 5(b)] and having the same charge $\left(\Phi_{2}^{\prime}=\Phi_{2}^{\prime \prime}\right)$. Nevertheless, the independence of these filaments allows them to have a small charge (and potential) difference $\delta \Phi \ll \Phi_{2}^{\prime}$. The obtained structure combines both above-considered DQWr structures: the horizontally and vertically designed ones. Of course, to provide $\delta \Phi / \Phi_{2}^{\prime} \ll 1$, the comparatively small distance, $2 w$, between the filaments $M_{2}^{\prime}$ and $M_{2}^{\prime \prime}$ should be large in comparison to the possible spatial error $\delta x$ of the position of the filament $M_{1}$ in relation to each of them.

If the distance $2 w$ is small in comparison to $z_{2}=z_{2}^{\prime}=z_{2}^{\prime \prime}$, the filament pair $M_{2}^{\prime}+M_{2}^{\prime \prime}$ can be considered as the single united filament with the doubled charge $\Phi_{2}=2 \Phi_{2}^{\prime}$. All the formulas written for the DQWr shown in Fig. 5(a) are also available for the one in Fig. 5(b). The single important distinction between them is that the latter [Fig. 5(b)] allows us to tune the S-AS splitting with the help of a small correcting charge difference $\delta \Phi \ll \Phi_{2}^{\prime}$.

\section{DISCUSSION: THE SIMPLEST SETUP}

To form electron devices on the basis of the aboveconsidered quantum wires and dots induced by potentials of initial metallic filamentlike gates require selecting (1) semiconductor $S$; (2) materials and structures of the metallic gate filaments; (3) materials and structures of dielectric layers, which form the combined barrier $B$; and (4) structure of the $B / S$ interface. An evident and attractive material for the role of the semiconductor $S$ is $\mathrm{Si}$, which has the natural partner, $\mathrm{SiO}_{2}$, for the role of the basic component for the barrier $B$. The main disadvantage of such a selection is a comparatively low value of the peak electron mobility $\mu_{s}$ in inversion and accumulation layers created at the $\mathrm{SiO}_{2} / \mathrm{Si}$ interface: 
$2.4 \mathrm{~m}^{2} / \mathrm{V} \mathrm{s}$ (Ref. 22), $1.5 \mathrm{~m}^{2} / \mathrm{V} \mathrm{s}$ (Ref. 23), $\leqslant 4.0 \mathrm{~m}^{2} / \mathrm{V} \mathrm{s}$ (Ref. 24), and $1.0 \mathrm{~m}^{2} / \mathrm{V} \mathrm{s}$ in thin Si layers grown between $\mathrm{SiO}_{2}$ layers. ${ }^{25}$ These values do not promise sufficiently large lifetimes of the electron states in our induced quantum wires and dots. The insufficiently large values of the mobility $\mu_{s}$ are explained by unavoidable roughness of the $\mathrm{SiO}_{2} / \mathrm{Si}$ interfaces and existence of bound charges ${ }^{22}$ in $\mathrm{SiO}_{2}$. As is known, the silicon dioxide is not the single dielectric material that can isolate metallic gates from $\mathrm{Si}$ surfaces. There exist the traditional competitors of $\mathrm{SiO}_{2}$, such as $\mathrm{SiO}_{x<2}$, $\mathrm{Si}_{3} \mathrm{~N}_{4}, \mathrm{SiO}_{x} \mathrm{~N}_{y}, \mathrm{Al}_{2} \mathrm{O}_{3}$, and also additionally $\mathrm{HfO}_{2}, \mathrm{ZrO}_{2}$ $\mathrm{TiO}_{2}$, etc., (see Ref. 26 as a review.) Unfortunately, we do not know the values of $\mu_{s}$ measured in the accumulation layers at $T \leqslant 4.2 \mathrm{~K}$ in the case of these gate dielectrics.

Another alternative to reach longer-lifetime-induced electron states is using Ge as the semiconductor $S$ instead of $\mathrm{Si}$. Germanium can be grown much cleaner and much more dislocation-free than silicon. For a long time, absence of the stable natural germanium dioxide $\mathrm{GeO}_{2}$ did not allow $\mathrm{Ge}$ to be a real silicon competitor even for low-temperature applications. Now, this situation can be changed as a result of the appearance of the other isolating gate dielectrics (for example, ${ }^{27-29}$ the same $\mathrm{ZrO}_{2}$ ). Unfortunately, we do not know the values of $\mu_{s}$ for $T \leqslant 4.2 \mathrm{~K}$ in this case as before, but they should be definitely higher than in the Si case.

The maximally tempting contenders for the role of the metallic gate filaments are both multiwall and single-wall carbon nanotubes (MW CNTs and SW CNTs). They combine high conductivities and the required very small transverse sizes. Now there appears a large number of communications on using CNTs as the active element (current-conducting channel) in field-effect transistors (FETs; see Ref. 30 as a review and Ref. 31.) As a rule, the silicon dioxide, $\mathrm{SiO}_{2}$, is used universally as a substrate, a top cover, and a gate dielectric in such FETs, but there are rare exceptions: for example, ${ }^{32} \mathrm{HfO}_{2}$. Though the problem of directed placement ${ }^{33}$ and directed fixation of nanotubes is far from the final solution, coating of SW CNTs with an isolating shell could be very progressive. There are several suggestions of coated nanotubes with the calibrated $\mathrm{SiO}_{2}$ shells (see Ref. 34 and references therein). Such coating of SW CNTs and their subsequent gluing together with the same silica can lead to multilayer structures containing the metallic (nanotube) gates on the top of the primary dielectric layer grown on the $\mathrm{Si}$ or Ge surface.

To foresee construction of complicated multielement systems (like quantum computing models) on the basis of the above-described QWrs, QDs, DQDs, etc., we should investigate parameters of the separate simplest elements and structures including a small number of such elements. Below, we consider two such elements: the induced $\mathrm{QWr}$ and quantum point contact (QPC). The layout shown in Fig. 6 is placed on the undoped semiconductor sample $S$ ( $\mathrm{Si}$ or $\mathrm{Ge}$ ) with two heavy doped regions: $n^{+}-S$ and $n^{+}-D$, contacting with an outer electric circuit that is out of the area of Fig. 6. The gates $M_{S}$ and $M_{D}$, placed on the top of the primary barrier $B_{1}$ and having sufficiently large sizes, are positively charged by potentials $V_{S}$ and $V_{D}$, respectively, and induce twodimensional electron gases (2DEGs) $2 \mathrm{DEG}_{S}$ and $2 \mathrm{DEG}_{D}$.

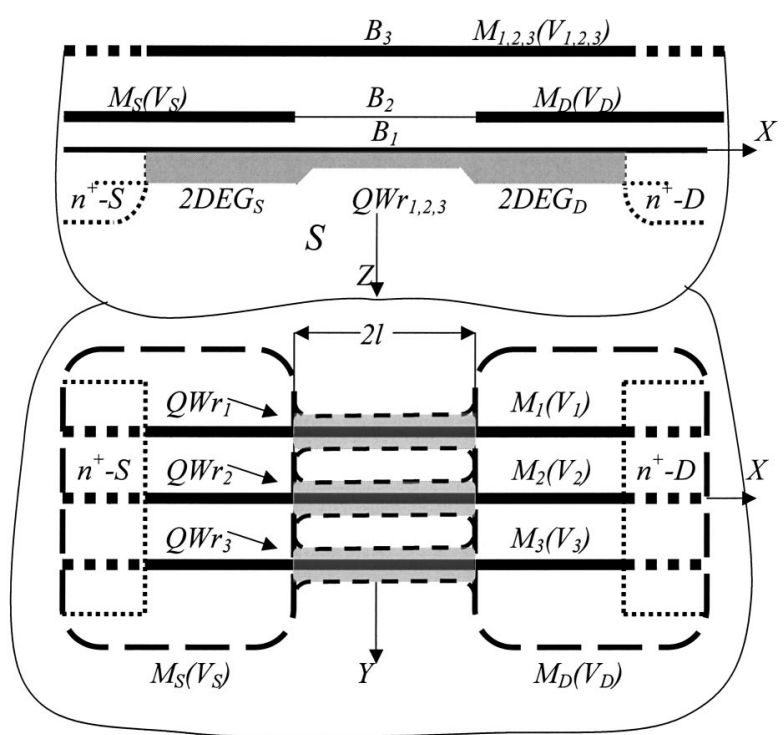

FIG. 6. Layout of the device for identification and measurement of the induced quantum wires.

These gases are separated from each other by a very narrow (substantially submicron) spatial interval $2 l \leqslant 0.1 \mu \mathrm{m}$. Several metallic filaments (we have three such filaments, $M_{1,2,3}$, in Fig. 6), which are isolated from the gates $M_{S, D}$ by the barrier $B_{2}$ and form the second level of the controlling system, intersect this spatial interval and induce the $\mathrm{QWrs}_{1,2,3}$. We have created the completely induced FETs, which are controlled both by the potentials $V_{S, D}$ and by the potentials $V_{1,2,3}$ of the filament gates $M_{1,2,3}$. The potentials $V_{S, D}$ form the controlled induced 2DEG source and drain and the potentials $V_{1,2,3}$ induce the QWr current-conducting channels. Currents in these channels should be measured as functions of both $V_{S, D}$ and $V_{1,2,3}$. The filament gates $M_{1,2,3}$ are fixed and buried by the barrier $B_{3}$. We lead outside the currentconducting contacts with the $n^{+}-S$ and $n^{+}-D$ heavily doped regions to measure the FET currents and contacts with the gates $M_{S, D}$ and $M_{1,2,3}$ to introduce the potentials $V_{S, D}$ and $V_{1,2,3}$. The subject of our measurements is the conductivities of each quantum wires $\mathrm{QWr}_{1,2,3}$, which depend on the abovementioned potentials. The potentials $V_{S, D}$ together with $V_{1,2,3}$ form configurations of the $2 \mathrm{D} / 1 \mathrm{D}$ contacts of the $2 \mathrm{DEG}_{S}$ and $2 \mathrm{DEG}_{D}$ to the $1 \mathrm{DEG}_{1,2,3}$ in the $\mathrm{QWr}_{1,2,3}$.

What do we expect as a result of such measurements? Since these measurements take place for low temperatures in the very short $\mathrm{QWr}_{1,2,3}(2 l \sim 0.1 \mu \mathrm{m})$, we should obtain ballistic electron transport and conductance quantization. In contrast to the AlGaAs/GaAs case, in which conductance quantization has been measured repeatedly both in different QPCs and in comparatively long QWrs, there are only several successful results for the Si case. ${ }^{35-41}$ Results in Refs. 35-38 have been obtained for very short metal-oxide semiconductor (MOS) structures, in which electron mobility $\mu_{s}$ is within the above-mentioned small values. ${ }^{22-25}$ The quantum wires in Refs. 39-41 have been induced in the tensilestressed 26-nm-thick $\mathrm{Si}$ layer grown on the undoped $\mathrm{Si}_{0.7} \mathrm{Ge}_{0.3}$ virtual (relaxed) substrate and covered by $\mathrm{Si}_{0.7} \mathrm{Ge}_{0.3}$ spacer followed by modulation donor doping layer and $\mathrm{Si}_{3} \mathrm{~N}_{4}$ dielectric. Appearance of the intermediate 

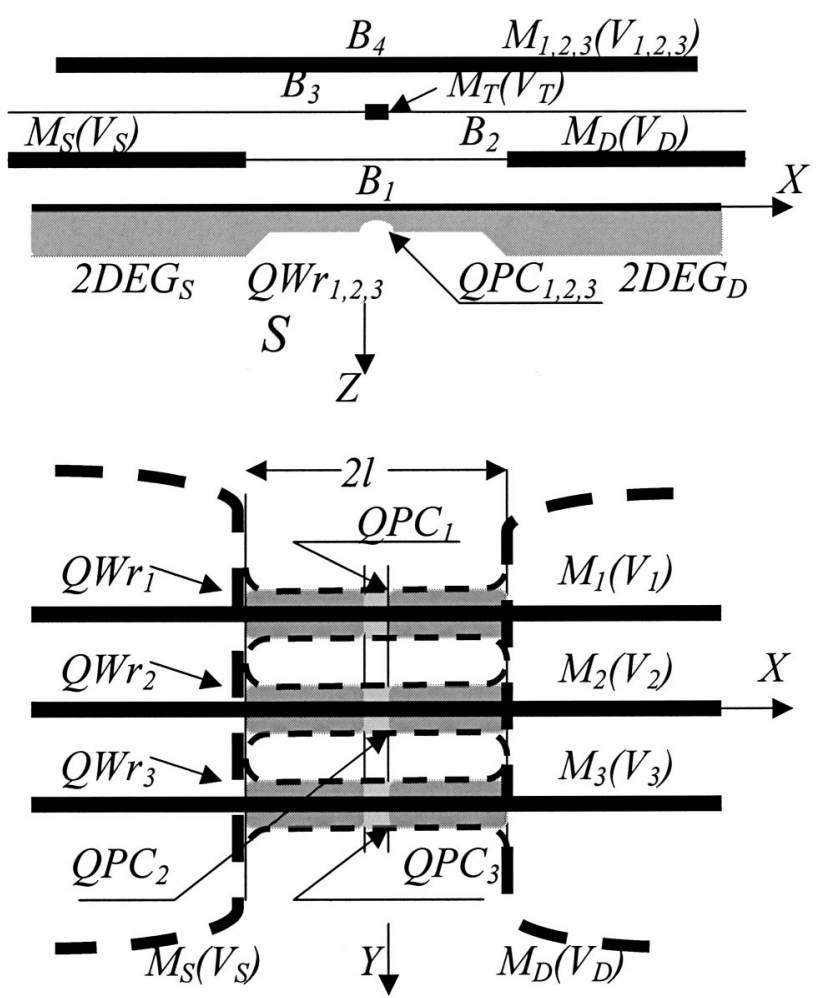

FIG. 7. Layout of the device for identification and measurement of the induced 1D/1D/1D-quantum point contacts.

$\mathrm{Si}_{0.7} \mathrm{Ge}_{0.3}$ layer between the active $\mathrm{Si}$ layer and the gate isolator substantially improves the 2DEG quality. The peak mobility value of $\mu_{s}\left(\sim 17.5 \mathrm{~m}^{2} / \mathrm{V} \mathrm{s}\right)$ in this case is much higher than in 2DEGs, induced directly under the $\mathrm{SiO}_{2} / \mathrm{Si}$ interface. Such mobility corresponds to the elastic mean free path $\sim 1.5 \mu \mathrm{m}$ that very much simplifies investigation for both QWr effects and, as a result, QD effects.

The potentials $V_{1,2,3}$ in the suggested structures in Fig. 6 determine the minimum width of our induced $\mathrm{QWr}_{1,2,3}$ and the potentials $V_{S, D}$ can change their effective length $2 l_{\text {eff }}$ $\leqslant 2 l$. If the initial length $2 l$ is insufficiently small, the effect of the positive accumulating potentials $V_{S, D}$ can be also insufficient to shorter $2 l_{\text {eff }}$ and make it shorter than the elastic mean free path $L_{e}$.

Below we suggest one more design of the induced OPC. It is illustrated in Fig. 7 where we show the middle part of the structure displayed in Fig. 6 with the added transverse filament gate $M_{T}$. We assume that this gate is charged by the negative potential $V_{T}<0$ and creates a certain electron depletion under it (like the filament $M_{1}$ in Fig. 5). This depleting filament interrupts the $\mathrm{QWrs}_{1,2,3}$ and forms depleted sections in the middle of them. These sections depend on both the potential $V_{T}$ and the potentials $V_{1,2,3}$ and are the actual OPCs. However, there is a noticeable difference between these OPCs and the traditional OPC. The traditional one is a 2D/ $1 \mathrm{D} / 2 \mathrm{D}$ structure, obtained on the basis of the 2DEG in a modulation-doped heterostructure. The above-considered QPC is a $1 \mathrm{D} / 1 \mathrm{D} / 1 \mathrm{D}$ structure, in which the middle $1 \mathrm{DEG}$ is depleted in comparison to the side ones. It is de facto a one-dimensional $n^{+} n n^{+}$conductor. It is a difficult problem to realize such a QPC by the traditional methods using modulation-doped heterostructures.
The same structure is shown in Fig. 7, but in the case of $V_{T}>0$, is suitable for formation of the intersection quantum dots considered in Sec. IV. Such QDs should appear under all the crossing points of the filament $M_{T}$ with the filament $M_{1,2,3}$. This charge exchange in the filament $M_{T}$ transforms the independent $\mathrm{QWr}_{1,2,3}$, shown in Figs. 6 and 7 , in the network connected by the new quantum wire $\mathrm{QWr}_{T}$ under this filament $M_{T}$. In this case, it is preferable to organize the additional second of current-conducting contacts analogous to $n^{+}-S$ and $n^{+}-D$ and the additional gate pair analogous to $M_{S, D}$ to provide current control in the $\mathrm{QWr}_{T}$. But such a four-contact bridgelike FET is outside of the frameworks of this article. We also bring out of these frameworks the induced FETs with the DQWr channels, which can be much more cumbersome in comparison to the layout shown in Fig. 6 [see for example, Ref. 42].

\section{CONCLUDING REMARKS}

Above we have theoretically substantiated the procedure of induction of quantum wires, quantum dots, doublequantum wires, double-quantum dots, and quantum point contacts in a semiconductor with the help of the charged metallic filaments embedded in the barrier space over the semiconductor in parallel to the barrier/semiconductor interface. These filaments can be placed on several levels over the primary $B / S$ interface to finally induce the complicated $2 \mathrm{D}$ device structure under the $B / S$ interface in the semiconductor. This structure should be controlled by the system of dc and ac potentials in the above-mentioned filaments and also by the potentials of the remote semiconductor $n^{+}$contacts and the potentials of the large gates assigned to these contacts (see the gates $M_{S, D}$ in Fig. 6). Such a gate system is able to provide a qubit control including entanglement and reading out processes.

We have assumed that it is possible to use $\mathrm{Si}$ and $\mathrm{Ge}$ as the basic semiconductor materials for our systems. The quality of the suggested structures is defined substantially by the $B / S$-interface quality. All of our wires and dots are attached by the gate potentials to this interface. Defects and roughnesses of this interface as well as the charged centers close to this interface can decrease the quality of the induced wires and dots very much. It seems that using a sufficiently thick layer of the tensile-stressed $\mathrm{Si}$ grown on the virtual $\mathrm{Si}_{0.7} \mathrm{Ge}_{0.3}$ substrate and separated from the barrier oxide by the moderately thick $\mathrm{Si}_{0.7} \mathrm{Ge}_{0.3}$ spacer could substantially improve qualities of the induced electron structures. In this case, the above-mentioned spacer would be a component of the thickened $B / S$ interface separating the dielectric barrier $B$ containing the metallic gates from the semiconductor $S$ (tensilestressed $\mathrm{Si}$ ) with the induced device structure.

\section{ACKNOWLEDGMENTS}

The authors thank Professor M. I. Dykman and Professor B. Golding from MSU and Dr. Z. D. Kwon from Novosibirsk Institute of Semiconductor Physics (Russia) for fruitful discussions. The work was supported by the Institute of Quantum Sciences at MSU and the AFOSR through the MURI program (Grant No. F 4960-00-0328.) 
${ }^{1}$ P. M. Morse and H. Feshbach, Methods of Theoretical Physics (Mc GrowHill, New York, 1953), Ch. 10.

${ }^{2}$ L. D. Landau and E. M. Lifshitz, Electrodynamics of Continuous Media (Pergamon, Oxford, NY, 1984).

${ }^{3}$ I. E. Tamm, Fundamentals of the Theory of Electricity (Mir, Moscow, 1964)

${ }^{4}$ M. Abramowitz and I. A. Stegun, Handbook of Mathematical Functions (Dover, New York, 1965).

${ }^{5}$ L. D. Landau and E. M. Lifshitz, Quantum Mechanics: Nonrelativistic Theory (Pergamon, Oxford, NY, 1965).

${ }^{6}$ R. L. Schult, D. G. Ravenhall, and H. W. Wyld, Phys. Rev. B 39, 5476 (1989).

${ }^{7}$ M. L. Roukes, A. Scherer, S. J. Allen, H. G. Craigheard, R. M. Ruthen, E. D. Beebe, and J. P. Harbison, Phys. Rev. Lett. 59, 3011 (1987).

${ }^{8}$ G. Timp, H. U. Baranger, P. de Vegvar, J. E. Cunningham, R. E. Howard, R. Behringer, and P. M. Mankiewich, Phys. Rev. Lett. 60, 2081 (1988).

${ }^{9}$ W. Wegscheider, G. Schedelbeck, G. Abstreiter, M. Rother, and M. Bichler, Phys. Rev. Lett. 79, 1917 (1997).

${ }^{10}$ W. Wegscheider, G. Schedelbeck, M. Bichler, and G. Abstreiter, Phys. Status Solidi A 164, 601 (1997).

${ }^{11}$ G. Schedelbeck, W. Wegscheider, M. Rother, S. Glutsch, M. Bichler, and G. Abstreiter, Physica E (Amsterdam) 2, 1 (1998).

${ }^{12}$ W. Wegscheider, G. Schedelbeck, M. Bichler, and G. Abstreiter, Physica E (Amsterdam) 3, 103 (1998).

${ }^{13}$ G. Schedelbeck, W. Wegscheider, M. Bichler, and G. Abstreiter, Science 278, 1792 (1997).

${ }^{14}$ H. Akiyama, J. Phys.: Condens. Matter 10, 3095 (1998).

${ }^{15}$ M. Grundmann and D. Bimberg, Phys. Rev. B 55, 4054 (1997).

${ }^{16}$ J. A. del Alamo, C. C. Eugster, Q. Hu, M. R. Melloch, and M. J. Rooks, Superlattices Microstruct. 23, 121 (1998).

${ }^{17}$ C. C. Eugster and J. A. del Alamo, Phys. Rev. Lett. 67, 3586 (1991).

${ }^{18}$ C. C. Eugster, J. A. del Alamo, M. J. Rooks, and M. R. Melloch, Appl. Phys. Lett. 64, 315 (1991).

${ }^{19}$ C. C. Eugster, J. A. del Alamo, M. R. Melloch, and M. J. Rooks, Phys. Rev. B 48, 15057 (1993).

${ }^{20}$ J. A. del Alamo and C. C. Eugster, Appl. Phys. Lett. 56, 78 (1990).

${ }^{21}$ N. Tsukada, A. D. Wieck, and K. Ploog, Appl. Phys. Lett. 56, 2527 (1990).
${ }^{22}$ T. Ando, A. B. Fowler, and F. Stern, Rev. Mod. Phys. 54, 437 (1982).

${ }^{23}$ S. B. Field, M. A. Kastner, U. Meirav, J. H. Scott-Thomas, D. A. Antoniadis, H. I. Smith, and S. J. Wind, Phys. Rev. B 42, 3523 (1990).

${ }^{24}$ V. M. Pudalov, M. E. Gershenson, H. Kojima, G. Brunthaler, A. Prinz, and G. Bauer, Phys. Rev. Lett. 91, 126403 (2003).

${ }^{25}$ K. Takashina, Y. Hirayama, A. Fujiwara, S. Horiguchi, and Y. Takahashi, Physica E (Amsterdam) 22, 72 (2004).

${ }^{26}$ C. K. Maiti, S. K. Samanta, S. Chatterjee, G. K. Dalapati, and L. K. Bera, Solid-State Electron. 48, 1369 (2004).

${ }^{27}$ C. O. Chui, B. B. Triplett, P. C. McIntyre, and K. C. Saraswat, IEEE Electron Device Lett. 23, 473 (2002).

${ }^{28}$ G. Tarashi, A. I. Pitera, and E. A. Fitzgerald, Solid-State Electron. 48, 1297 (2004).

${ }^{29}$ D. Chi, C. O. Chui, K. C. Saraswat, B. B. Triplett, and P. C. McIntyre, J. Appl. Phys. 96, 813 (2004).

${ }^{30} \mathrm{Ph}$. Avouris, J. Appenzeller, R. Martel, and S. J. Wind, Proc.-IEEE Ultrason. Symp. 91, 1772 (2003).

${ }^{31}$ J. A. Misevich, R. Martel, Ph. Avouris, J. C. Tsang, S. Heinze, and J. Tersoff, Science 300, 783 (2003).

${ }^{32}$ J. Appenzeller, J. Knoch, R. Martel, V. Derycke, S. J. Wind, and Ph. Avouris, IEEE Trans. Nanotechnol. 1, 184 (2002).

${ }^{33}$ L. A. Nagahara, I. Amlani, J. Lewenstein, and R. K. Tsui, Appl. Phys. Lett. 80, 3826 (2002).

${ }^{34}$ E. A. Whitsitt and A. R. Barron, Nano Lett. 3, 775 (2003).

${ }^{35}$ K. Takeuchi and R. Newbury, Phys. Rev. B 43, 7324 (1991).

${ }^{36}$ S. L. Wang, P. C. van Son, B. J. van Wees, and T. M. Klapwijk, Phys. Rev. B 46, 12873 (1992).

${ }^{37}$ K. Nishiguchi and S. Oda, Appl. Phys. Lett. 76, 2922 (2000).

${ }^{38}$ K. Nishiguchi and S. Oda, J. Appl. Phys. 92, 1399 (2002).

${ }^{39}$ M. Holzmann, D. Többen, G. Abstreiter, W. Wendel, H. Lorenz, J. P. Kotthaus, and F. Schäffler, Appl. Phys. Lett. 66, 833 (1995).

${ }^{40}$ D. Többen, D. A. Wharam, G. Abstreiter, J. P. Kotthaus, and F. Schäffler, Semicond. Sci. Technol. 10, 711 (1995).

${ }^{41}$ M. Holzmann, D. Többen, P. Baumgartner, G. Abstreiter, A. Kriele, H. Lorenz, and F. Schäffler, Surf. Sci. 361/362, 673 (1996).

${ }^{42}$ Z. S. Gribnikov, A. N. Korshak, and V. V. Mitin, J. Appl. Phys. 83, 1481 (1998). 